each two nonparallel elements of $G$ cross each other. Obviously the conclusions of the theorem do not hold.

The following example will show that the condition that no two elements of the collection $G$ shall have a complementary domain in common is also necessary. In the cartesian plane let $M$ be a circle of radius 1 and center at the origin, and $N$ a circle of radius 1 and center at the point $(5,5)$. Let $G_{1}$ be a collection which contains each continuum which is the sum of $M$ and a horizontal straight line interval of length 10 whose left-hand end point is on the circle $M$ and which contains no point within $M$. Let $G_{2}$ be a collection which contains each continuum which is the sum of $N$ and a vertical straight line interval of length 10 whose upper end point is on the circle $N$ and which contains no point within $N$. Let $G=G_{1}+G_{2}$. No element of $G$ crosses any other element of $G$, but uncountably many have a complementary domain in common with some other element of the collection. However, it is evident that no countable subcollection of $G$ covers the set of points each of which is common to two continua of the collection $G$.

It is not known whether or not the condition that each element of $G$ shall separate some complementary domain of every other one can be omitted.

Oklahoma Agricultural and Mechanical College

\title{
A PRINCIPAL AXIS TRANSFORMATION FOR NON-HERMITIAN MATRICES
}

\section{CARL ECKART AND GALE YOUNG}

The availability of the principal axis transformation for hermitian matrices often simplifies the proof of theorems concerning them. In working with non-hermitian matrices (square or rectangular) it was found that a generalization of this transformation has a similar use for them.* A special case of this generalization has been investigated by Sylvester $\dagger$ who proved Theorem 1 (below) for square matrices with real elements. The unitary matrices $U$ and $V$ are in that case orthogonal matrices with real elements. Special cases had also been

* C. Eckart, The kinetic energy of polyatomic molecules, Physical Review, vol. 46 (1934), p. 383; C. Eckart and G. Young, The approximation of one matrix by another of lower rank, Psychometrika, vol. 1 (1936), p. 211; A. S. Householder and G. Young, Matrix approximation and latent roots, American Mathematical Monthly, vol. 45 (1938), p. 302.

† Sylvester, Messenger of Mathematics, vol. 19 (1889), p. 42. 
discussed earlier by Beltrami and Jordan, and more recently Autonne and $\mathrm{E}$. T. Browne have proved the theorem for square matrices with complex elements. $\dagger$

The following definitions will be convenient for the present purpose. An $(r, s)$ matrix is one having $r$ rows and $s$ columns; its elements may be complex numbers. The hermitian transpose of an $(r, s)$ matrix $A$, whose elements are $a_{i j}$, is the $(s, r)$ matrix $A^{*}$ whose elements are $\left(a^{*}\right)_{j i}=\bar{a}_{i j}$. An $(r, s)$ matrix is diagonal if its elements $a_{i j}$ are all zero unless $i=j$.

THEOREM 1. For every $(r, s)$ matrix $A$, there are two unitary matrices $U$ and $V$, such that

$$
D=U^{*} A V
$$

is a diagonal matrix with real elements, none of which are negative.

The proof of this theorem may be based on the observation that $A A^{*}$ is a non-negative definite hermitian $(r, r)$ matrix; for it is the Gram matrix of the rows of $A$, considered as vectors. Consequently there are $r$ vectors (that is, $r(r, 1)$ matrices) $X_{i}$ such that

$$
A A^{*} X_{i}=d_{i}^{2} X_{i}
$$

and

$$
X_{i}^{*} X_{k}=\delta_{i k}, \quad i, k=1, \cdots, r .
$$

The numbers $d_{i}{ }^{2}$ are the characteristic values of $A A^{*}$, and the $X_{i}$ are unit vectors along its principal axes. The numbers $d_{i}$ are real and may be defined to be nonnegative. It is convenient to arrange the numbering of these vectors so that

$$
d_{1} \geqq d_{2} \geqq \cdots \geqq d_{n}>0, \quad d_{n+1}=\cdots=d_{r}=0 .
$$

In the same way, there are $s$ vectors (that is, $s(s, 1)$ matrices) $Y_{j}$ such that

$$
A^{*} A Y_{j}=e_{j}^{2} Y_{j}
$$

and

$$
Y_{j}^{*} Y_{l}=\delta_{j l}
$$

If

$$
e_{1} \geqq e_{2} \geqq \cdots \geqq e_{m}>0, \quad e_{m+1}=\cdots=e_{s}=0,
$$

$\dagger$ Autonne, Sur les matrices hypohermitiennes et les unitaires, Comptes Rendus de l'Académie des Sciences, Paris, vol. 156 (1913), pp. 858-860; E. T. Browne, this Bulletin, vol. 36 (1930), p. 707. 
it can be shown that $m=n$ and $e_{i}=d_{i}$ whenever $i \leqq n$. For if $X_{i}$ are the vectors of (1), (2), and (3), then the $n$ vectors defined by

$$
Y_{i}=A^{*} X_{i} / d_{i}
$$$$
i \leqq n,
$$

will satisfy (4) and (5) with $e_{i}=d_{i}$. Since the characteristic values are unique, it follows that $m$ cannot be less than $n$; inverting the argument, we see that $n$ cannot be less than $m$.

Any set of vectors $X_{i}$ for which (1), (2), and (3) hold may be considered as the columns of a unitary $(r, r)$ matrix $U$. Then let (6) define the first $n$ columns of an $(s, s)$ matrix $V$, and fill in the remaining columns to make $V$ unitary. These matrices $U$ and $V$ then satisfy the requirements of the theorem.

To prove this we may first observe that if $D$ is the matrix $U^{*} A V$, then $D D^{*}=U^{*} A A^{*} U$ is a diagonal matrix with diagonal elements $d_{i}{ }^{2}$, and $D^{*} D=V^{*} A^{*} A V$ is a diagonal matrix with diagonal elements $e_{i}^{2}$. Furthermore, if the matrix $D$ is written as

$$
D=\left\|\begin{array}{ll}
D_{1} & D_{2} \\
D_{3} & D_{4}
\end{array}\right\|,
$$

where $D_{1}, D_{2}, D_{3}$, and $D_{4}$ are $(n, n),(n, s-n),(r-n, n)$, and $(r-n, s-n)$ matrices, respectively, then these properties of $D D^{*}$ and $D^{*} D$ imply

$$
D_{3} D_{3}^{*}+D_{4} D_{4}^{*}=0, \quad D_{2}^{*} D_{2}+D_{4}^{*} D_{4}=0
$$

(among other equations). Since $D_{2}^{*} D_{2}, D_{3} D_{3}^{*}$, and $D_{4} D_{4}^{*}$ are all nonnegative definite hermitian matrices, it follows that they are all null matrices, and from this, that $D_{2}, D_{3}$, and $D_{4}$ are all null matrices. It remains to be shown that $D_{1}$ is diagonal with no negative elements. Its $i j$-element may be written $X_{i}^{*} A Y_{j}$; and from (6), (1), and (2) it readily follows that

$$
X_{i}^{*} A Y_{j}=d_{j} \delta_{i j}, \quad i, j \leqq n,
$$

which completes the proof of Theorem 1.

The above also proves the following result:

Corollary. In Theorem 1, $U^{*}$ may be any unitary matrix which diagonalizes $A A^{*}$, and there then exists a unitary matrix $V$ such that the theorem is true. Similarly, $V^{*}$ may be taken as any unitary matrix which diagonalizes $A^{*} A$, and there then exists a matrix $U$ satisfying the requirements of the theorem.

The theorem on the simultaneous transformation of two hermitian matrices to principal axes also generalizes. 
TheOREM 2. If $A$ and $B$ are both $(r, s)$ matrices, then there are two unitary matrices $U$ and $V$ such that $E=U^{*} A V$, and $F=U^{*} B V$ are both diagonal matrices with real elements and such that $E$ has no negative elements, if and only if $A B^{*}$ and $B^{*} A$ are both hermitian matrices.

The necessity of the condition is an immediate consequence of the invariance of the class of hermitian matrices under transformations of the form $U C U^{*}$ when $U$ is unitary, for $E F^{*}$ is hermitian and $A B^{*}=U E F^{*} U^{*}$, and so on. The sufficiency may be proved as follows. Because of Theorem 1, it is no loss of generality to suppose that $A$ has already been transformed to the form

$$
\left\|\begin{array}{ll}
D & O_{2} \\
O_{3} & O_{4}
\end{array}\right\|
$$

where $D$ is a real diagonal $(n, n)$ matrix of rank $n$, having no negative elements, and $O_{2}, O_{3}, O_{4}$ are null matrices. The matrix $B$ may be divided into corresponding submatrices:

$$
\left\|\begin{array}{ll}
G & K \\
L & H
\end{array}\right\|
$$

Then the condition of the theorem leads to

$$
K=O_{2}, \quad L=O_{3}, \quad D G^{*}=G D, \quad G^{*} D=D G .
$$

In element notation, the last two equations are

$$
d_{i} \bar{g}_{j i}=d_{j} g_{i j}, \quad d_{j} \bar{g}_{j i}=d_{i} g_{i j} .
$$

Since $d_{i}, d_{j}>0$, it readily follows that $G$ is hermitian and that $D G=G D$. From the theorem on the simultaneous transformation of two hermitian matrices to principal axes, it follows that there is a unitary $(n, n)$ matrix $P$ such that $P^{*} D P=D$ and $P^{*} G P$ is diagonal with real elements. From Theorem 1, it follows that there are also two unitary matrices $Q$ and $R$ such that $Q^{*} H R$ is a diagonal matrix with real elements. It is then readily seen that the matrices

$$
U=\left\|\begin{array}{ll}
P & O_{3}{ }^{*} \\
O_{3} & Q
\end{array}\right\|, \quad V=\left\|\begin{array}{cc}
P & O_{2} \\
O_{2}{ }^{*} & R
\end{array}\right\|
$$

will satisfy Theorem 2 .

The authors desire to acknowledge helpful discussion with Professor G. A. Bliss and Dr. Saunders MacLane.

The University of Chicago 